\title{
MPC for battery/fuel cell hybrid vehicles including fuel cell dynamics and battery performance improvement
}

\author{
Alicia Arce, Alejandro del Real and Carlos Bordons \\ Departamento de Sistemas y Automtica, Universidad de Sevilla, Spain
}

\begin{abstract}
This paper presents a hybrid MPC control for PEM fuel cell vehicle. The main goals of such controller are battery performance improvement while taking into account operational modes such as charge control, continuous discharge and step discharge; and the inclusion of time constraints in switching on and off the fuel cell. The problem proposed, which have been implemented successfully, implies handling with continuous and discrete variables and constraints. Regarding control strategy, the controller tracks motor power demand and keeps batteries close to the state of discharge which is adequately chosen to obtain most efficient performance. Moreover,as an innovation, nonlinear and linear models of the vehicle have been developed including fuel cell dynamics which are important for this control level and particularized for a laboratory prototype in order to allow a future real implementation of the hybrid control design. Finally, simulation results are illustrated in several figures which depict the control performance for different driving cycles and initial conditions.
\end{abstract}

$\overline{1}$ A.Arce, A.J del Real and C. Bordons are with the Departamento de Ingeniera de Sistemas y Automtica, Universidad de Sevilla, Escuela Superior de Ingenieros, Camino de 


\section{Introduction}

Fuel cells have been considerably developed the last decade because of the social energy concern. Such dispositive is considered as a good candidate to replace in the future the conventional energy systems provoking an energy revolution in stationary and mobile applications. The scope of this paper is the automotive application [1]. Accordingly with [2], vehicles can not be driven only by fuel cells. Such kind of systems needs an energy storage system to support fuel cell during start-ups and acceleration peaks while storing energy during regenerative braking. There are many papers which analyze storage system based on conventional batteries, remarking Lithium-ion batteries [3] and ultracapacitors. Batteries generally have high energy density but less power density than ultracapacitors. The principal weaknesses of this technology are calendar and cycle life and cost. The system studied in this paper includes lithium-ion batteries as energy storage system.

Studying state-of-the-art control power management of fuel cell hybrid vehicles, papers such as [4-6] apply several advance control technics to regulate power management efficiently . However, none of them implement control algorithms contemplating different battery operational modes to improve the performance and cycle life. In regards to vehicle modeling, all the works design and simulate controllers on quasi-static models eliminating fuel cell dynamics.

This work is based on a previous heuristic control which minimizes the hydrogen consumption keeping battery state of discharge over a optimal value as well. This heuristic algorithm [7] tackles with around 40 different dynamics states. However,

los Descubrimientos s/n. 41092 Sevilla, Spain, e-mail:aarce,adelreal@cartuja.us.es, bordons@esi.us.es 
if battery modes are included, the number of states is too high to implement it robustly. Therefore, advance control technics are required. Hybrid control theory [8], which has been implemented previously in hybrid electric vehicles (HEV) because handling with discrete variables, is one of the best alternatives for such applications as commented in [2]. Hybrid control theory has been successfully applied in fuel cell-renovable energy resources applications [9].In such applications, discrete variables are included in order to constrain fuel cell switching times and number of auxiliary devices start-up.

The main goal of this paper is the implementation of a hybrid controller for vehicle power management while considering different battery operation modes such as charge, continuous discharge and discontinuous discharge and constraining fuel cell start-up and shutdown. Consequently, battery performance is improved.

The paper is organized as follows: in section II, the real system is described briefly; in section III, the non-linear vehicle model is presented and in section IV, the linear model used for the control design is explained. In section $\mathrm{V}$, the hybrid control model is formulated and section VI presents the hybrid based-model predictive controller. Controller behaviors are tested for several simulations in section VII. Finally, the conclusions and the future works are discussed in section VIII.

\section{System Description}

This paper studies the system which scheme is sketched in fig 1.Particularly the system is a vehicle propelled by an hybrid system based on PEM fuel cells and batteries. All the components are also showed on the scheme where is remarkable the topology of the prototype under development [1], which includes two different 
DC to DC converters. One of both rises and regulates the voltage supplied by the fuel cell to the DC bus voltage and the other one rises and reduces the battery voltage depending on the battery mode (charge or discharge) to the DC bus voltage. Also, there is another DC to AC converter before the motor. The design of each component has been done to meet the minimum requirements for passenger cars [4]. The following figure shows the main components of the hybrid vehicle.

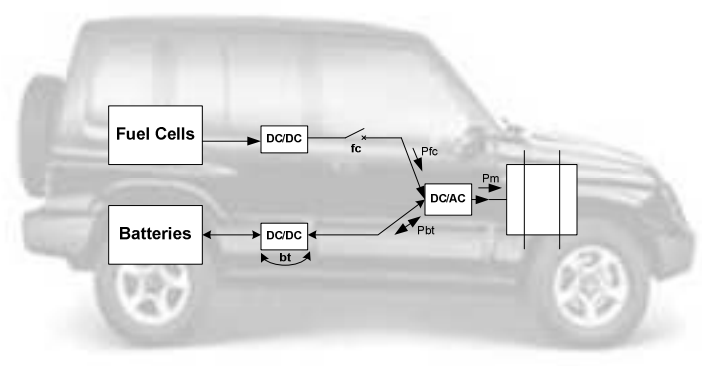

Fig. 1. Scheme of the hybrid vehicle

Components resulting from vehicle design step are:

- Polymeric Electrolyte Membrane fuel cell; this kind of fuel cell has been chosen because of the fast start-up and the low operational temperatures. Furthermore, the maximum fuel cell power for the prototype is $56 \mathrm{~kW}$, the number of cells are 256 and the output fuel cell voltage range is $150-247 \mathrm{~V}$.

- Lithium-ion Batteries are excellent candidates for these applications since the specific energy is very high. For the purpose of meeting the design specifications, eight modules of this sort of batteries are stacked in serie in the prototype. The characteristics of an individual module are enumerated in table 1 
Table 1

Lithium-ion battery characteristics

\begin{tabular}{|c|c|}
\hline Characteristics & Values \\
\hline Nominal voltage & $19.2 \mathrm{~V}$ \\
\hline Capacity (C/5) & $65 A h$ \\
\hline Specific energy & $84 W h / k g$ \\
\hline Energy density & $117 \mathrm{Wh} / l$ \\
\hline \multicolumn{2}{|c|}{ Standard Discharge $23^{\circ} \mathrm{C}$} \\
\hline Maximum continuous current & $125 A$ \\
\hline Maximum current $30 \mathrm{sec}$ pulse & $220 A$ \\
\hline Cut-off voltage & $15 \mathrm{~V}$ \\
\hline \multicolumn{2}{|l|}{ Standard Charge } \\
\hline Charge voltage & $21.9 \mathrm{~V}$ \\
\hline Maximum charge current & $30 A$ \\
\hline Charge time & $2.5 \mathrm{hrs}$ \\
\hline
\end{tabular}

- Permanent Magnet Synchronous Motor (PMSM) is coupled to the back steering by direct coupling on the same shaft. Specifically The maximum motor angular speed is 5976r.p.m, the maximum motor power is $66 \mathrm{~kW}$ and the maximum torque is $460 \mathrm{Nm}$. This unit works as a generator during regenerative braking and as a motor the rest of the time. The power losses in both cases are different.

- The Hydrogen tank meets the European rules for explosive fuel in vehicles. for the sake of obtaining a $100 \mathrm{~km}$ autonomy and taking into account that the pressure inside the tank is $350 \mathrm{bar}$, the capacity is $2.4 \mathrm{~kg}$ of $\mathrm{H}_{2}$. 


\section{Non-linear model}

The non-linear model is necessary to analysis the control problem and to simulate the results of the controller. The model is quasi-static and combines the equations of all the components being enough for the control level proposed in this paper, as done by [4].Furthermore, fuel cell dynamics which are remarkable in the control level studied have been included innovating on previous models based on [4].

The model is divided in two parts; the first one models the vehicle taking as an input the driving cycle and as an output the power demanded by the motor. The other part considers the power generation, that is battery and fuel cell models, and the inputs are the power demanded to the fuel cell and to the battery and the outputs are the motor power supplied, the hydrogen consumption and the battery state of discharge. The controller must regulate the power generation satisfying the motor power demand for a driving cycle. The model is sketched in fig 2 .

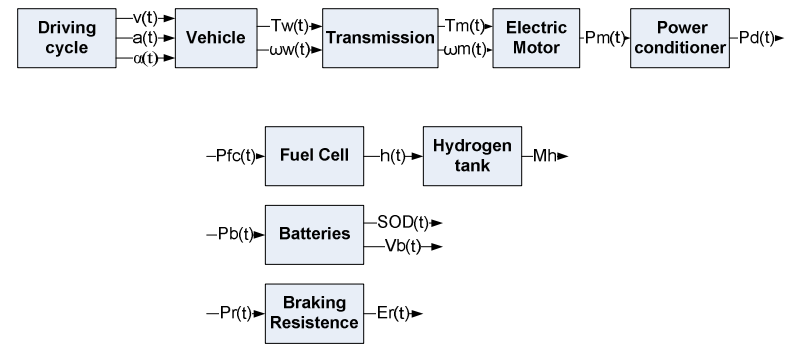

Fig. 2. Scheme model; vehicle and power generation

The equations of each component are described in the next subsections.

Driving Cycle.- The speed profile was gathered from the program Advisor [10] and it depends on the place where the car was driven, for instance, the driving cycle for a city road is totally different than the one for a highway. The vehicle acceleration 
is obtained from the vehicle speed using the following equation.

$$
a=\frac{d v(t)}{d t}
$$

Also, the model requires the slope profile of the path. Due to the difficulty of gathering such data, the path is assumed to be completely flat, so that the slope angle is $0^{\circ}$ for all the driving cycle.

Vehicle.- The block called vehicle as showed in fig 2 calculates motor torques and motor angular speeds from driving cycle data for the specific characteristics of the car design (weight... etc). The equations are:

$$
F_{t}(t)=m_{v} \cdot a_{v}(t)+F_{a}(t)+F_{r}(t)+F_{g}(t)
$$

Where $F_{t}$ is the traction force on the wheels, $m_{v}$ is the vehicle mass, $a_{v}$ is the linear vehicle acceleration, $F_{a}$ is the aerodynamic friction, $F_{r}$ is the rolling friction and $F_{g}$ is the force caused by gravity.

$$
\begin{gathered}
F_{a}(v)=\frac{1}{2} \cdot \rho_{a} \cdot A_{f} \cdot c_{d} \cdot v^{2} \\
F_{r}=c_{r} \cdot m_{v} \cdot g \cdot \cos (\theta), v>0 \\
F_{g}(\alpha)=m_{v} \cdot g \cdot \sin (\theta)
\end{gathered}
$$

where $c_{d}$ is the aerodynamic coefficient that varies with many factors. For the sake of simplicity, it is considered constant. $A_{f}$ is the frontal vehicle area, $\rho_{a}$ is the density of the ambient air, $c_{r}$ is the rolling friction coefficient, $g$ is the acceleration due the gravity and $\theta$ is the slope.

$$
T_{w}(t)=F_{t}(t) \cdot r_{w}, \quad w_{w}(t)=\frac{v(t)}{r_{w}}
$$

$T_{w}$ is the traction torque on the wheels, $\omega_{w}$ is the wheel angular speed and $r_{w}$ is the wheel radius. 
Transmission.- The coupling as commented in the previous section is direct and on the same shaft. The gear ratio, $\gamma$, is figured out from the design data. This ratio has been calculated when the vehicle speed is $150 \mathrm{~km} / \mathrm{h}$ and the motor angular speed is 6000 r.p.m.

$$
w_{e}=\frac{\gamma \cdot v(t)}{r_{w}}
$$

where $w_{e}$ is the motor angular speed and $\gamma$ is the gear ratio which is equal to 5.427.

$$
T_{e}(t)=\frac{T_{w}(t)}{\gamma}, \quad w_{e}(t)=w_{w}(t) \cdot \gamma
$$

where $T_{e}$ is the motor torque and $w_{e}$ is the motor angular speed.

Electric Motor.- This model calculates power profiles demanded by the electric motor in order to track driving cycles. As expounded in previous sections, the unit works as generator while braking and as motor the rest of the time. The power is expressed as:

$$
P_{e}=T_{e}(t) \cdot w_{e}(t)
$$

Regarding the losses during the motor mode:

$$
\begin{aligned}
& W_{e}>2000 \mathrm{rpm} P_{l}=0.016 \cdot T_{e}^{2}+0.0082 \cdot w_{e}^{1.5} \\
& W_{e} \leq 2000 \mathrm{rpm} P_{l}=4.05 \cdot 10^{-9} \cdot T_{e}^{2} \cdot w_{e}^{2}+0.0082 \cdot w_{e}^{1.5}
\end{aligned}
$$

For the case of the motor working as a generator, the losses are:

$$
P_{m}=P_{e} \cdot \mu
$$

Assuming the losses ratio $\mu$ constant and equal to 0.8 , the total power demanded by the motor is computed from this equation:

$$
P_{m}=P_{e}+P_{l}
$$


Power Conditioner.- The study fulfilled in this paper involves the losses caused by these devices. The model is assumed static since the dynamics are over two or three times more than the rest. The efficiency is set at 0.95 .

$$
P_{b u s, D C}=\frac{P_{m}}{\mu_{c}}
$$

Batteries The battery model is based on [11] and simplified neglecting the losses due to each component performance. Moreover, this model includes thermal battery dynamics.

$$
\begin{gathered}
i(t)=\frac{P_{b}(t)}{v(t) \cdot n_{\text {battery }}} \\
E(i(t), T(t), t)=v(i(t), T(t), t)-R_{\text {int }} \cdot i(t) \\
v(i(t), T(t), t)=n_{\text {battery }} \cdot \sum_{k=0}^{n} c_{k} \cdot S O D^{k}(i(t), T(t), t)+\Delta E(T) \\
S O D(i(t), T(t), t)=S O D_{0}+\frac{1}{Q_{r}} \int_{0}^{t} \alpha(i(t)) \cdot \beta(T(t)) \cdot i(t) d t
\end{gathered}
$$

where $E(i(t), T(t), t)$ is the equilibrium potential, $R_{\text {int }}$ is the internal resistor, $v$ is the battery voltage and $i(t)$ is the battery current, which is positive whether the battery is discharging and negative in the case of charge. $\Delta E(T)$ is a coefficient which compensates the variation of the equilibrium potential due to the temperature, $\alpha(i(t))$ compensates the variations on the discharge curve (this curve represents the discharge voltage against the state of discharge) caused by the current and $\beta(T(t))$ corrects the discharge curve deviation due to the temperature. $Q_{r}$ is the battery capacity, $\operatorname{SOD}(i(t), T(t), t)$ is the state of discharge (the state of charge is equal to $1-S O D)$ and $n_{\text {battery }}$ is the number of modules connected.

$$
\begin{aligned}
m \cdot c_{p} \cdot \frac{d T(t)}{d t}= & i(t)^{2} \cdot R_{1}+\frac{1}{R_{2}}(v(t)+E(i(t), T(t), t)+ \\
& \left.+i(t) \cdot R_{1}\right)^{2}-h_{c} \cdot A \cdot\left(T(t)-T_{a}\right)
\end{aligned}
$$

where $m$ is the battery mass, $c_{p}$ is the specific heat, $R_{1}$ and $R_{2}$ are internal resistor, 
$h_{c}$ heat transfer coefficient, $A$ battery external surface area and $T_{a}$ is the ambient temperature.

PEM Fuel Cell.- The fuel cell model, which is a simplification of the model presented in [12], includes the internal dynamics. As seen in fig 2, the fuel cell block output is the hydrogen consumption, which is indirectly minimized with the control strategy proposed.

$$
i_{s t}(t)=\frac{P_{f c}(t)}{v_{s t}(t)}, \quad h(t)=m_{H_{2}} \cdot n \cdot \frac{i_{s} t(t)}{2 \cdot F}, \quad v_{s t}(t)=f\left(i_{s t}\right)
$$

$i_{s t}(t)$ is the stack current, $v_{s t}(t)$ is the stack voltage, $m_{H_{2}}$ is the hydrogen molar mass, $n$ is the number of cells, $\mathrm{F}$ is the Faraday coefficient and $f$ is the polarization curve.

The characteristic time of the fuel cell model particularized for this application is $200 \mathrm{~ms}$, so that the next equation includes this dynamic to the model. The fuel cell dynamics which has been taken into account in this control level are due to the air flow dynamic.

$$
P_{f c}+0.2 \cdot \frac{d P f c}{d t}=P_{d e m}
$$

where $P_{d e m}$ is the fuel cell power demanded by the system and $P_{f c}$ is the power supplied by the fuel cell to the system.

Hydrogen tank.- The tank is modeled by the following equation:

$$
m(t)=m_{0}-\int_{0}^{t} h(s) d s
$$

Braking Resistors.- This block calculates the amount of energy which the system 
wastes during brakes.

$$
E_{\text {wasted }}=\int_{0}^{t} P_{\text {resistors }}(s) d s
$$

The particularization of all the equations listed for the vehicle design are detailed in [7].

\section{Linear Model}

In order to design the controller, the system linearization is essential. The linear system resulted from the non-linear equation presented in the previous section is showed in fig 3 . The linear model works with power approach instead of current approach as a result of the topology of the case in study fig 1, although working with currents facilitates the linearization [13]. In this topology, the batteries are connected to the DC bus incorporating a DC to DC converter contrasting with [13] which eliminates the converter. Considering a DC to DC converter requires an extra variable to relate the battery voltage, which is not available. Hence, the linearization is much more complicated since the system involves divisions and is highly nonlinear. Fig 3 shows the linear model scheme, where are displayed the inputs and the outputs. The simplified nonlinear fuel cell model is presented by the equations 20

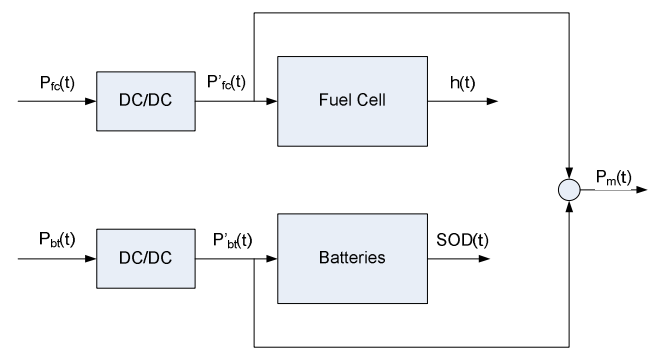

Fig. 3. Control Scheme 
and the linearization is illustrated in fig 4, being the linear model:

$$
\frac{d h}{d t}=1.3843 \cdot 10^{-2} \cdot P_{f c}
$$

Fig 4 represents the fuel cell polarization curve and the hydrogen consumption curves.
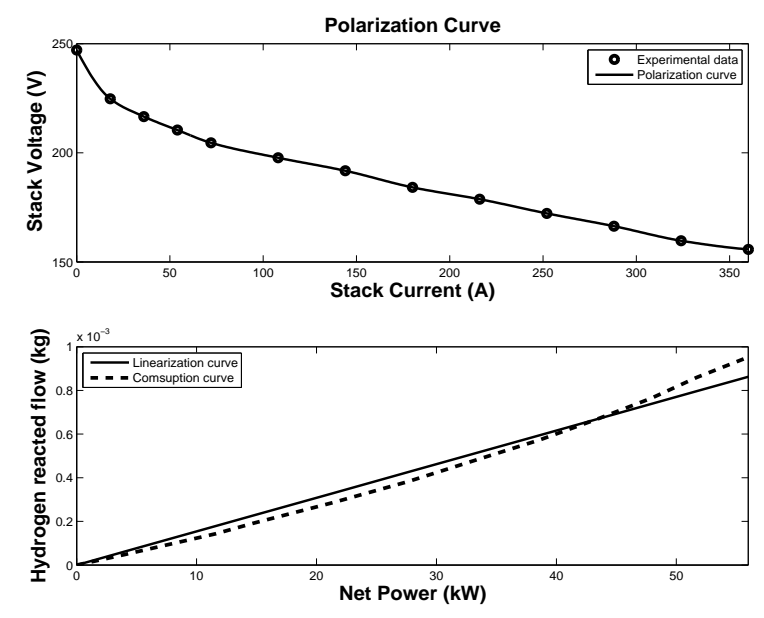

Fig. 4. Fuel Cell Current Linearization

Regarding the battery linearization, the thermal dynamics are neglected. Therefore, the thermal coefficients are constant. The equations 16-18 are linearized as:

$$
\begin{gathered}
S O D=4.2735 \cdot 10^{-6} \cdot \int_{0}^{t} i(t) d t \\
v_{b}=154.9-6.914 \cdot 10^{-5} \cdot \int_{0}^{t} i(t) d t
\end{gathered}
$$

The comparison of the non-linear and linear battery model is plotted in fig 5

As discussed previously, the equation 15 is highly nonlinear. A Piece-Wise Affine (PWA) model is obtained to reduce the error and the linearization has been done around three different operational points depending on battery powers. 

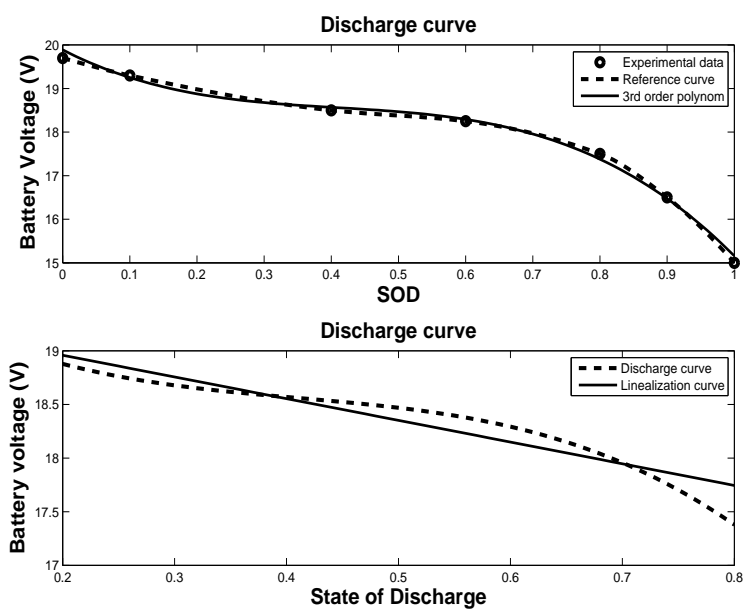

Fig. 5. Battery voltage Linearization

$$
\begin{aligned}
P_{b} \leq 0.5 i_{b}(t) & =0.6824+6.812 \cdot P_{b}(t)-0.00464 \cdot v_{b}(t) \\
5 \geq P_{b}>0.5 & i_{b}(t)=6.824+6.812 \cdot P_{b}(t)-0.0464 \cdot v_{b}(t) \\
P_{b}>5 & i_{b}(t)=68.244+6.812 \cdot P_{b}(t)-0.4645 \cdot v_{b}(t)
\end{aligned}
$$

The following illustration shows a comparison of the nonlinear model with the model linearized. The results have been simulated with the same initial conditions (the initial state of discharge is an essential parameter that influences the simulation results) and with the same inputs, which are represented in fig 3. The inputs have been taken from an heuristic controller [14], which is the background of the hybrid controller.

The charge controller is integrated in the linear model. There are two ways to adequately charge the lithium-ion batteries as commented in [15] and [16]. The constant-current/ constant-voltage $(\mathrm{CC} / \mathrm{CV})$ method is the fastest and suitable for automobile applications, which involve fast charges during regenerative brakes. This method supplies constant current (approximately the current for 1C) to the batteries until reaching the maximum voltage and the current is decreased, this point is reached when the batteries are charged over $85 \%$, which is the upper limit imposed by the controller. The charge current for this particular case is $30 \mathrm{~A}$ and the 

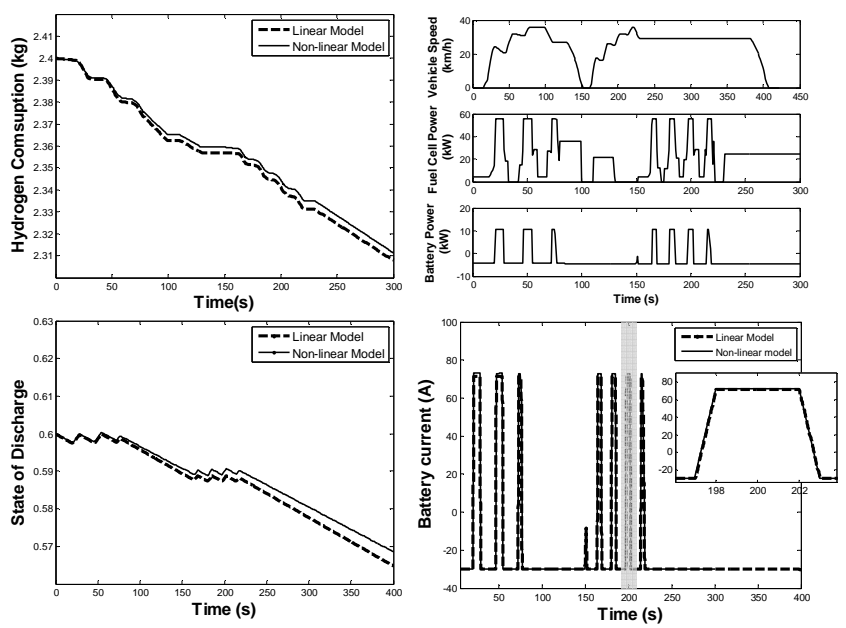

Fig. 6. Comparison of linear system with nonlinear system

model control equation is:

$$
v_{b}(t)=-4.695+0.485376 \cdot S O D
$$

After the linearization, the next step is the discretization of the model. The characteristic time of the system corresponds with the reaction time of the vehicle driver, which is around $1 s$. Thus, the sampling time chosen is 0.02

\section{Hybrid Control Model}

As has been said, the hybrid systems framework [17], [18] is adopted to describe the process, due to the presence of both continuos and binary inputs. Although many modeling formalisms can be found to describe hybrid systems [19], the mixedlogical (MLD) formulation [20] is adopted here. The purpose of the hybrid system description language [8] is the procurement of MLD models from a high-level textual description of hybrid dynamics. Accordingly, four main blocks will be considered (as shown in Fig 7) along with the system constraints. Each of these blocks, their nomenclature and their functionality are described in the following subsec- 
tions.

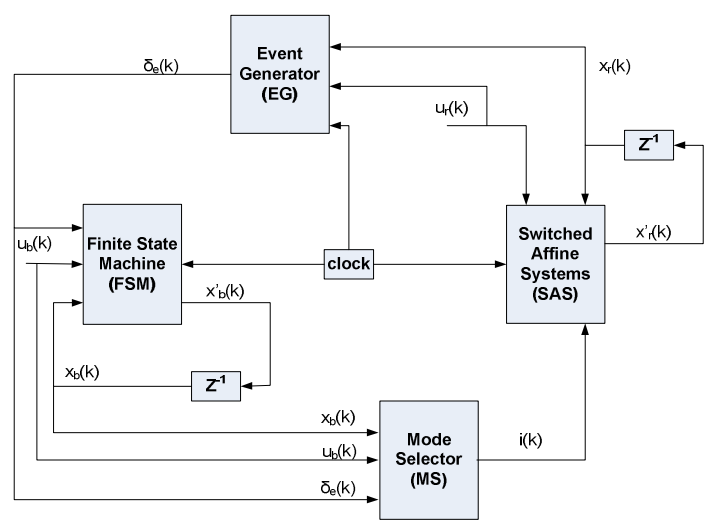

Fig. 7. Hybrid modeling conception

\subsection{Switched Affine System}

As described in [8], a switched affine system is a collection of linear affine systems:

$$
\begin{aligned}
x_{r}(k+1) & =A_{i(k)} x_{r}(k)+B_{i(k)} u_{r}(k)+f_{i(k)} \\
y_{r}(k) & =C_{i(k)} x_{r}(k)
\end{aligned}
$$

where $\mathrm{k} \in \mathbb{Z}^{+}$is the time indicator, $x_{r} \in \mathfrak{X}_{r} \subseteq \mathbb{R}^{n_{r}}$ is the exogenous continuous input vector, $y_{r} \in \mathfrak{Y}_{r} \subseteq \mathbb{R}^{p_{r}}$ is the continuous output vector, $\left\{A_{i}, B_{i}, f_{i}, C_{i}\right\}$ is a collection of matrices of suitable dimensions, and the mode $i_{k} \in \mathfrak{I}$ is an input signal that chooses the linear state update dynamics.

Let $x_{r}=\left[\begin{array}{lllllll}P_{m}(k) & h(k) & S O D(k) & i_{b}(k) & t_{1}(k) & t_{2}(k) & t_{3}(k)\end{array}\right]^{T}, y_{r}(k)=\left[\begin{array}{llll}P_{m}(k) & h(k) & S O D(k)\end{array}\right]^{T}$ and $u_{r}=\left[\begin{array}{ll}P_{b}(k) & P_{f c}\end{array}\right]$. The matices $\left\{A_{i}, B_{i}, f_{i}, C_{i}\right\}$ are defined as follows: 


$$
A_{i(k)}=\left(\begin{array}{ccccccc}
0 & 0 & 0 & 0 & 0 & 0 & 0 \\
0 & 1 & 0 & 0 & 0 & 0 & 0 \\
0 & 0 & 1 & 4.273 \cdot 10^{-6} \cdot T_{s} & 0 & 0 & 0 \\
0 & 0 & 0 & a_{44} & 0 & 0 & 0 \\
0 & 0 & 0 & 0 & a_{55} & 0 & 0 \\
0 & 0 & 0 & 0 & 0 & a_{66} & 0 \\
0 & 0 & 0 & 0 & 0 & 0 & a_{77}
\end{array}\right), B_{i(k)}=\left(\begin{array}{cc}
1 & 1 \\
0 & b_{22} \\
0 & 0 \\
b_{41} & 0 \\
0 & 0 \\
0 & 0 \\
0 & 0
\end{array}\right), f_{i(k)}=\left(\begin{array}{c}
0 \\
0 \\
0 \\
f_{4} \\
f_{5} \\
f_{6} \\
f_{7}
\end{array}\right)
$$

Table 2, 3 and 4 show the values of the parameters, $a_{44}, a_{55}, a_{66}, a_{77}, b_{22}, b_{41}, f_{4}, f_{5}, f_{6}, f_{7}$ depending on the input signals $i_{k}$, which are included in the appendix. Lastly,

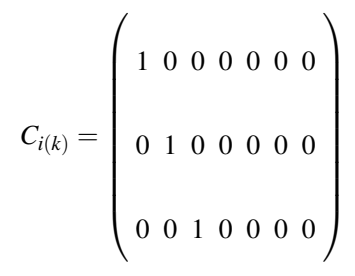

\subsection{Event Generator}

An event generator is a mathematical object that generates a logic signal according to the satisfaction of a linear affine constraint [8]:

$$
\delta_{e}(k)=f_{H}\left(x_{r}(k), u_{r}(k), k\right)
$$

where $f_{H}: \mathbb{R}^{n_{r}} \times \mathbb{R}^{m_{r}} \times \mathbb{Z}_{\geq 0} \rightarrow \mathfrak{D} \subseteq\{0,1\}^{n_{e}}$ is a vector of descriptive functions of a linear hyperplane, and $\mathbb{Z}_{\geq 0} \triangleq\{0,1, \ldots\}$ is the set of nonnegative integers. In particular, time events are modeled as $\left[\delta_{e}^{i}(k)=1\right] \leftrightarrow\left[a^{T} x_{r}(k)+b^{T} u_{r}(k) \leq c\right]$ where the superscript $i$ denotes the $\mathrm{i}$-th component of a vector.

Concerning the model proposed, the events $\delta_{e}(k)$, which depend on fuel cell startups and also fuel cell power supplied, are the following conditions: 


$$
\begin{aligned}
& \delta_{e}^{1}=1 \quad \text { if } \quad P_{f c} \leq 0.3 \quad \text { or } 0 \quad \text { otherwise } \\
& \delta_{e}^{2}=1 \quad \text { if } \quad P_{f c} \leq 0 \quad \text { or } 0 \quad \text { otherwise } \\
& \delta_{e}^{3}=1 \quad \text { if } \quad t_{1} \geq 5 \quad \text { or } 0 \quad \text { otherwise } \\
& \delta_{e}^{4}=1 \quad \text { if } \quad t_{2} \geq 25 \quad \text { or } 0 \quad \text { otherwise }
\end{aligned}
$$

In order to model the different modes of the batteries such as step discharge, continuous discharge and charge, is necessary include the events listed below:

$$
\begin{aligned}
& \delta_{e}^{5}=1 \quad \text { if } \quad P_{b} \leq 0 \quad \text { or } 0 \quad \text { otherwise } \\
& \delta_{e}^{6}=1 \quad \text { if } \quad P_{b} \geq 0 \quad \text { or } 0 \quad \text { otherwise } \\
& \delta_{e}^{7}=1 \quad \text { if } \quad i_{b} \leq 125 \quad \text { or } 0 \text { otherwise } \\
& \delta_{e}^{8}=1 \quad \text { if } \quad i_{b} \geq 125 \quad \text { or } 0 \text { otherwise } \\
& \delta_{e}^{9}=1 \quad \text { if } \quad t_{3} \leq 60 \quad \text { or } 0 \text { otherwise } \\
& \delta_{e}^{10}=1 \quad \text { if } \quad t_{3} \geq 30 \quad \text { or } 0 \text { otherwise }
\end{aligned}
$$

Regarding the charge control, the events are:

$$
\begin{array}{llllll}
\delta_{e}^{11}=1 & \text { if } & P_{b} \leq \text { chargecontrol } & \text { or } & 0 & \text { otherwise } \\
\delta_{e}^{12}=1 & \text { if } & P_{b} \geq \text { chargecontrol } & \text { or } & 0 & \text { otherwise }
\end{array}
$$

where chargecontrol is a linear variable which calculates the battery power demanded during the charge according with the $\mathrm{CC} / \mathrm{CV}$ charge method. This variable is calculated by the equation 30 .

\subsection{Finite State Machine (FSM)}

According with [8], a finite state machine (or automata) is a discrete dynamic process that evolves depending on a logic state update function:

$$
x_{b}^{\prime}(k)=f_{B}\left(x_{b}(k), u_{b}(k), \delta_{e}(k)\right)
$$


where $x_{b} \in \mathrm{X}_{b} \subseteq\{0,1\}^{n_{b}}$ is the Boolean state, $u_{b} \in \mathrm{U}_{b} \subseteq\{0,1\}^{m_{b}}$ is the exogenous input coming from the $\mathrm{EG}$, and $f_{B}: \mathrm{X}_{b} \times \mathrm{U}_{b} \times \mathrm{D} \rightarrow \mathrm{X}_{b}$ is a deterministic logic function.

Particularly, the system has four boolean states $\left\{x_{b 1}, x_{b 2}, x_{b 3}, x_{b 4}\right\}$, which are related with the four operation modes of the hybrid vehicle. The possible cases when the vehicle is working are: the fuel cell is off and the batteries are supplying the power demanded; the fuel cell is off and the batteries are charging with the power generated during the regenerative braking; the fuel cell is on and the batteries are charging with the power that the fuel cell is supplying; and lastly, the fuel cell is on and the batteries also are supplying power in peaks. All these cases are represented in the hybrid model in this way:

$$
\begin{aligned}
& x_{b 1}=\neg f c \wedge \neg b t \\
& x_{b 2}=\neg f c \wedge b t \\
& x_{b 3}=f c \wedge b t \\
& x_{b 4}=f c \wedge \neg b t
\end{aligned}
$$

where $\neg$ is the logic operation Not, $\wedge$ corresponds with the logic operation And and lastly $\vee$ is the logic operation Or.

\subsection{Mode Switch Selector}

Citing [8], the logic state $x_{b}(k)$, the boolean inputs $u_{b}(k)$, and the events $\delta_{e}(k)$ select the dynamic mode $i(k)$ of the switched affine system through a boolean function $f_{M}: \mathfrak{X}_{b} \times \mathfrak{U} \times \mathfrak{D} \rightarrow \mathfrak{I}$, which is therefore called mode selector. The output of this function,

$$
i(k)=f_{M}\left(x_{b}(k), u_{b}(k), \delta(k)\right)
$$


is called active mode. $A$ mode switch occurs at step $k$ if $i(k) \neq i(k-1)$. Note that in this discrete-time setting, a mode switch can only occur at sampling instants, contrary to the case of continuous time hybrid models.

Considering the boolean inputs $u_{b}(k)$ to be the on-off fuel cell signal $f c$ and the charge-discharge battery signal $b t$, in this application there are thirty-four different active modes, which are next summarized:

$$
\begin{aligned}
& i=1 \ldots i+2 \ldots 13 \text { if } \mathrm{x}_{\mathrm{b} 1} \wedge \delta_{\mathrm{e}}^{5} \wedge \delta_{\mathrm{e}}^{6} \wedge\left(\delta_{\mathrm{e}}^{8} \vee \neg \delta_{\mathrm{e}}^{8}\right) \wedge \\
& \left(\delta_{\mathrm{e}}^{9} \vee \neg \delta_{\mathrm{e}}^{9}\right) \wedge\left(\delta_{\mathrm{e}}^{10} \text { or } \neg \delta_{\mathrm{e}}^{10}\right) \\
& i=2 \ldots i+2 \ldots 14 \text { if } \mathrm{x}_{\mathrm{b} 1} \wedge\left(\delta_{\mathrm{e}}^{5} \vee \delta_{\mathrm{e}}^{6}\right) \wedge\left(\delta_{\mathrm{e}}^{8} \vee \neg \delta_{\mathrm{e}}^{8}\right) \wedge \\
& \left(\delta_{\mathrm{e}}^{9} \vee \neg \delta_{\mathrm{e}}^{9}\right) \wedge\left(\delta_{\mathrm{e}}^{10} \vee \neg \delta_{\mathrm{e}}^{10}\right) \\
& i=15 \text { if } \mathrm{x}_{\mathrm{b} 1} \wedge\left(\delta_{\mathrm{e}}^{5} \vee \delta_{\mathrm{e}}^{6}\right) \wedge \neg \delta_{\mathrm{e}}^{8} \wedge \delta_{\mathrm{e}}^{9} \\
& \wedge \delta_{\mathrm{e}}^{10} \\
& i=16 \text { if } \mathrm{x}_{\mathrm{b} 1} \wedge\left(\delta_{\mathrm{e}}^{5} \vee \delta_{\mathrm{e}}^{6}\right) \wedge \neg \delta_{\mathrm{e}}^{8} \wedge \\
& \left(\neg \delta_{\mathrm{e}}^{9} \vee \neg \delta_{\mathrm{e}}^{10}\right) \\
& i=17 \text { if } \mathrm{x}_{\mathrm{b} 2} \\
& i=18 \text { if } \mathrm{x}_{\mathrm{b} 3} \\
& i=19 \ldots i+2 \ldots 31 \text { if } \mathrm{x}_{\mathrm{b} 4} \wedge \delta_{\mathrm{e}}^{5} \wedge \delta_{\mathrm{e}}^{6} \wedge\left(\delta_{\mathrm{e}}^{8} \vee \neg \delta_{\mathrm{e}}^{8}\right) \wedge \\
& \left(\delta_{\mathrm{e}}^{9} \vee \neg \delta_{\mathrm{e}}^{9}\right) \wedge\left(\delta_{\mathrm{e}}^{10} \vee \neg \delta_{\mathrm{e}}^{10}\right) \\
& i=20 \ldots i+2 \ldots 32 \text { if } \mathrm{x}_{\mathrm{b} 4} \wedge\left(\delta_{\mathrm{e}}^{5} \vee \delta_{\mathrm{e}}^{6}\right) \wedge\left(\delta_{\mathrm{e}}^{8} \vee \neg \delta_{\mathrm{e}}^{8}\right) \wedge \\
& \left(\delta_{\mathrm{e}}^{9} \vee \neg \delta_{\mathrm{e}}^{9}\right) \wedge\left(\delta_{\mathrm{e}}^{10} \vee \neg \delta_{\mathrm{e}}^{10}\right) \\
& i=33 \text { if } \mathrm{x}_{\mathrm{b} 4} \wedge\left(\delta_{\mathrm{e}}^{5} \vee \delta_{\mathrm{e}}^{6}\right) \wedge \neg \delta_{\mathrm{e}}^{8} \wedge \delta_{\mathrm{e}}^{9} \\
& \wedge \delta_{\mathrm{e}}^{10} \\
& i=34 \text { if } \mathrm{x}_{\mathrm{b} 4} \wedge\left(\delta_{\mathrm{e}}^{5} \vee \delta_{\mathrm{e}}^{6}\right) \wedge \neg \delta_{\mathrm{e}}^{8} \wedge \\
& \left(\neg \delta_{\mathrm{e}}^{9} \vee \neg \delta_{\mathrm{e}}^{10}\right)
\end{aligned}
$$

\subsection{Constraints}

In this section, the system constraints are discussed. Moreover, an important part of this work is focused on the battery constraints including charge control, continuous discharge and step discharge. The constraint in the number of fuel cell start-ups 
and the constraint in the time between start-ups and shutdowns of the fuel cell are included to avoid fast switchings. All the constraints are formulated according with [8] as follows:

$$
\begin{aligned}
S O D & \geq 0.2 \\
S O D & \leq 0.8 \\
P_{f c} & \geq 0 \\
P_{f c} & \leq 56 \\
P_{b} & \geq-4.7 \\
i_{b} & \leq 220 \\
\delta_{e}^{1} & \rightarrow \neg f c \\
\neg \delta_{e}^{1} & \rightarrow f c \\
\neg f c & \rightarrow \delta_{e}^{2} \\
\delta_{e}^{6} & \rightarrow \neg b t \\
\neg \delta_{e}^{6} & \rightarrow b t \\
x_{b 2} \vee x_{b 3} & \rightarrow \delta_{e}^{11} \wedge \delta_{e}^{12} \\
\neg f c & \leftarrow\left(x_{b 1} \vee x_{b 2}\right) \wedge \neg \delta_{e}^{3} \\
f c & \leftarrow\left(x_{b 3} \wedge x_{b 4}\right) \vee \neg \delta_{e}^{4} \\
\delta_{e}^{7} & \leftarrow \delta_{e}^{10} \wedge \delta_{e}^{9} \wedge \delta_{e}^{8}
\end{aligned}
$$

\section{Hybrid model-based predictive controller (MPC) with constraints}

Hybrid MPC formulation has been successfully applied to many industrial applications. In this approach, at each sampling instant, a finite horizon open-loop optimization problem is solved, assuming the current state to be the initial condition for the problem. Based on the receding horizon philosophy [21], only the first element of the control sequence obtained is applied to the hybrid system. The same process is subsequently repeated at each sampling time, providing a feedback which allows disturbance rejection and reference tracking. Thus, the control problem is formulated as: 


$$
\begin{aligned}
\min _{\{u\}_{0}^{N-1}} J(u, x)= & \sum_{k=0}^{N}\left(\left\|\mathrm{Q}_{\mathrm{x}}\left(x_{k}-x_{r e f}\right)\right\|+\left\|\mathrm{Q}_{\mathrm{y}}\left(y_{k}-y_{r e f}\right)\right\|\right)+ \\
& +\left\|\mathrm{Q}_{\mathrm{xT}}\left(x(N \mid t)-x_{r}\right)\right\|
\end{aligned}
$$

subject to the simplified plant model and its constraints, where $N$ is the control horizon, $x(t)$ is the state of the MLD system at time $t$ and $R$ and $Q$ are weight matrices [20].

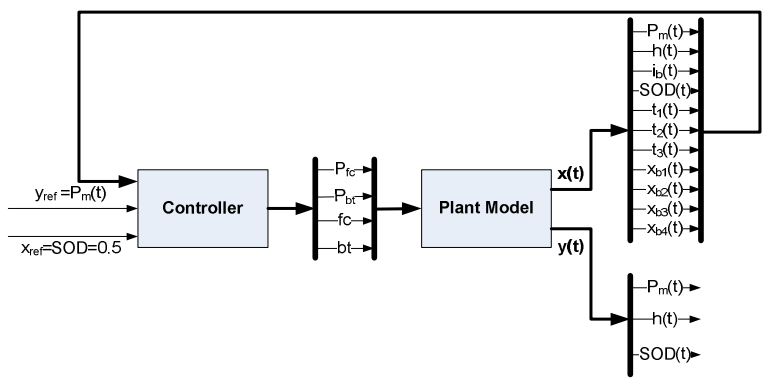

Fig. 8. Control Scheme

In this application, a $T_{s}=0.02$ sampling time as expounded in section 4 , a control horizon of $N=3$ and two references are considered as shown in fig 8 . The $y_{\text {ref }}$ is set to be $P_{m}$, which is the desired motor power in $k W$. A state reference is also added, where $x_{\text {ref }}=S O D=0.5$ (that is, aiming to keep the battery state of discharge around the point of maximum performance). This, in conjunction with the selected weight matrices, provides a weighted, multi-objective MPC controller, whose main objective is to track the output reference $y_{r e f}$, while at the same time utilizing the battery power in order to minimize hydrogen consumption. The resulting weight matrices $Q_{x}, Q_{x T}$ and $Q_{y}$ are: 


$$
\begin{gathered}
Q_{x}:=\left[q x_{i, j}\right]_{11 \times 11}=\left\{\begin{array}{l}
q x_{i, j}=30 \text { for } i=j=4 \\
q x_{i, j}=0 \quad \forall i, j / i=j \neq 4
\end{array}\right. \\
Q_{x T}:=\left[q x T_{i, j}\right]_{11 \times 11}=\left\{\begin{array}{l}
q x T_{i, j}=300 \text { for } i=j=4 \\
q x T_{i, j}=0 \quad \forall i, j / i=j \neq 4
\end{array}\right. \\
Q_{y}:=\left[q y_{i, j}\right]_{3 \times 3}=\left\{\begin{array}{l}
q y_{i, j}=0.9 \text { for } i=j=1 \\
q y_{i, j}=0 \quad \forall i, j / i=j \neq 1
\end{array}\right.
\end{gathered}
$$

\section{Simulation results}

In this section, the controller results are depicted in several graphs for different simulations. Firstly, a driving cycle was chosen to show the battery step mode as represented in fig 9 . The initial battery state of discharge is essential in the analysis. In fig 9 , the initial state of discharge is 0.3 , in other words, the batteries are initially almost full-charged. The subplot titled 'Battery Current' shows how the controller performs for a battery current upper of $125 \mathrm{~A}$. Notice that the battery can only supply power in step mode of 30 seconds because of the thermal constraints. In addition, the regenerative brake wasted power is also impoartant. As justified in the motor power subplot, the power reference is not completely tracked during regenerative brakes since the charge controller constraints the amount of power taken.

Fig 10 depicts the battery charge control when the fuel cell charges the batteries and during regenerative brakes. The driving cycle is the same as in fig 9 but the initial state of discharge is 0.7 , that is, the batteries are close to the full-discharge 

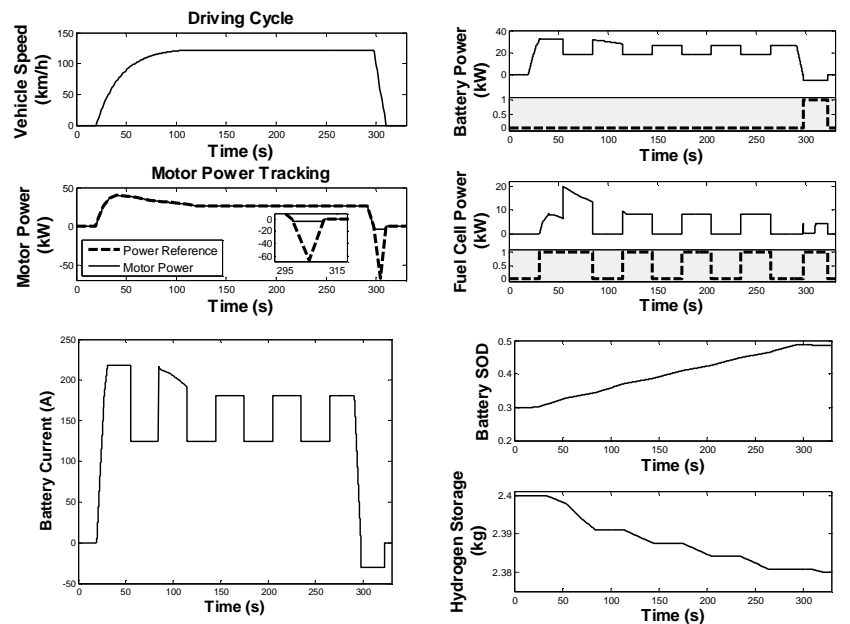

Fig. 9. Step Mode Battery

limitation. The performance of the charge power regulated by the charge controller included in the hybrid model can be observed in the battery power subplot. Notice that the power references are properly tracked by the controller.Also, the fuel cell which has to supply the necessary power to meet the motor demand plus the charge power demanded by the battery charge controller.
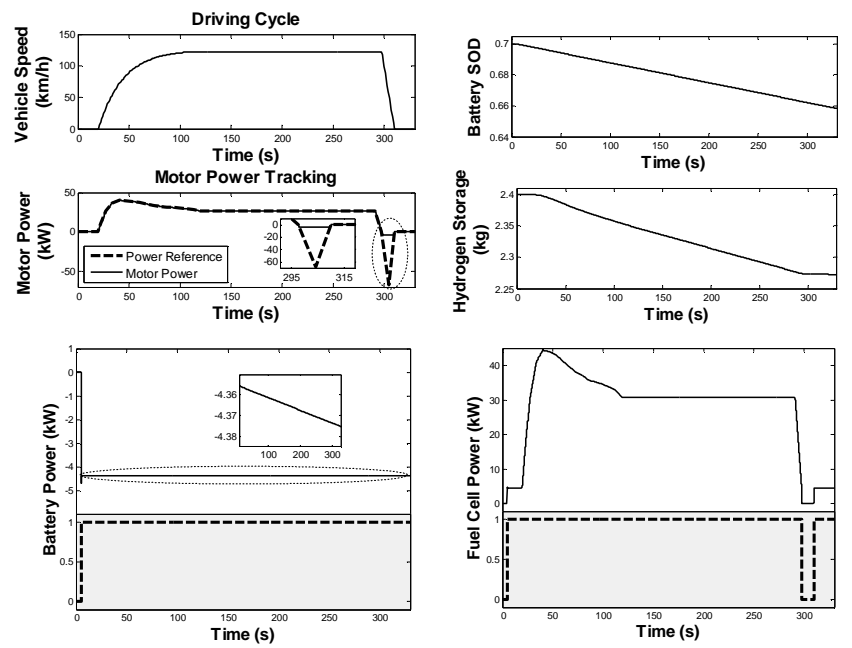

Fig. 10. Step Mode Battery

A simulation which mixes the three alternative battery modes is presented in fig 11 . The constraint of the time which the fuel cell is off is reduced in order to show how the controller performs. Emphatically, the motor power demand is not satisfaced 
because the fuel cell is shut down due to the time constraint described before and the demand is higher than the maximum battery power supplied.
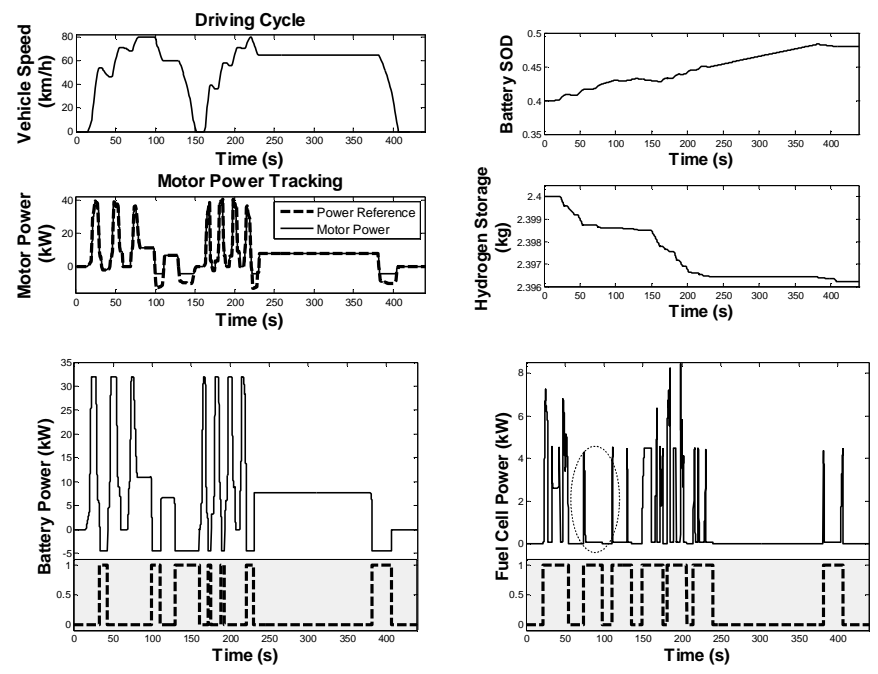

Fig. 11. Step Mode Battery

The time the fuel cell must be working is also constrained, as illustrated in fig 11. A power threshold is set around $300 \mathrm{~W}$, that is, the minimum fuel cell power demand which the controller considers to keep the fuel cell on. If the controller tries to shut down the fuel cell to improve the performance but the fuel cell-on time is not fulfilled, the controller set the fuel cell power demand on the threshold. This issues are clarified in the fuel cell power subplot 11.

Finally, fig 12 shows the effects of the fuel cell dynamics. The controller demands more power to the fuel cell to compensate the dynamics.

\section{Conclusion}

This paper has presented an hybrid concept for the generation power control in PEM fuel cell vehicles. The hybrid control optimization includes dicrete variables such as the fuel cell on-off and the battery charge-discharge. Furthermore, discrete 


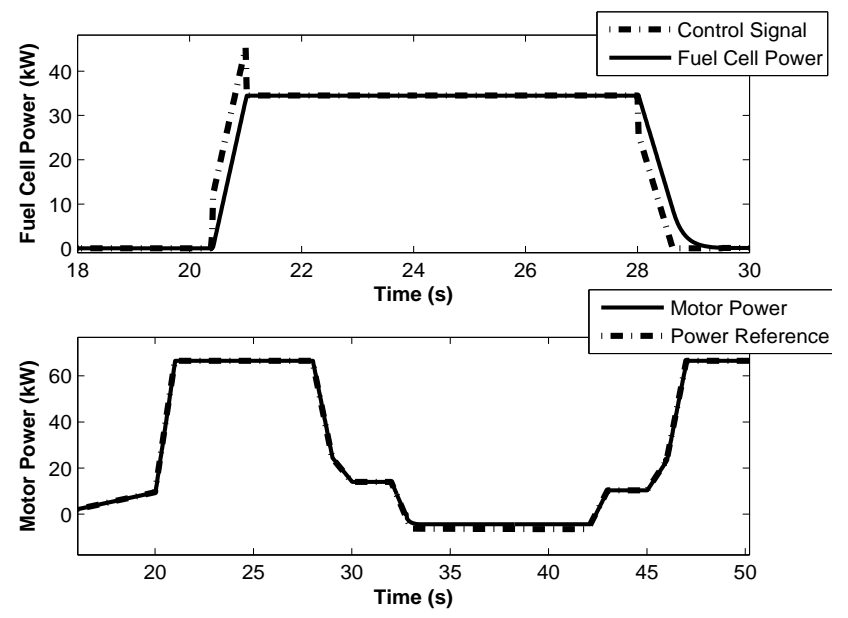

Fig. 12. Fuel cell dynamics

constraints have been taken into account in this analysis in order to improve the battery efficiency. The results are promising and the inclusion of start-up dynamic is an open issue for future works. The control objectives are the power tracking and the state of discharge control around a fixed value. The last reference could be changed depending on the driving cycle increasing the global efficiency and vehicle autonomy. Due to this reason stochastic predictions of driving cycle are been currently studied.

Moreover, given that the system sampling time is critic $(20 \mathrm{~ms})$, explicit hybrid formulation is an issue for future research, reducing the computational time implementing the optimization off-line [22].

\section{ACKNOWLEDGMENTS}

The authors would like to acknowledge the support of prof. A. Stefanopoulou from the Fuel Cell Systems Laboratory, University of Michigan. 


\section{Appendix}

Table 2

Parameter values

\begin{tabular}{|c||c|c|c|}
\hline & $i=1, i+2,13$ & $i=2, i+2,14$ & $i=15$ \\
\hline \hline$a_{44}$ & $-3.2116 \cdot 10^{-7} \cdot T_{s}$ & 0 & $-3.2116 \cdot 10^{-7} \cdot T_{s}$ \\
\hline$a_{55}$ & 1 & 1 & 1 \\
\hline$a_{66}$ & 0 & 0 & 0 \\
\hline$a_{77}$ & 1 & 1 & 0 \\
\hline$b_{22}$ & 0 & 0 & 0 \\
\hline$b_{41}$ & 6.8125 & 0 & 6.8125 \\
\hline$f_{4}$ & -0.03707 & 0 & -0.03707 \\
\hline$f_{5}$ & $T s$ & $T s$ & $T s$ \\
\hline$f_{6}$ & 0 & 0 & 0 \\
\hline$f_{7}$ & $T_{s}$ & $T_{s}$ & 0 \\
\hline
\end{tabular}


Table 3

Parameter values

\begin{tabular}{|c||c|c|c|c|}
\hline & $i=16$ & $i=17$ & $i=18$ & $i=19, i+2,31$ \\
\hline \hline$a_{44}$ & 0 & 0 & 0 & $-3.2116 \cdot 10^{-6} \cdot T_{s}$ \\
\hline$a_{55}$ & 1 & 1 & 1 & 0 \\
\hline$a_{66}$ & 0 & 0 & 1 & 1 \\
\hline$a_{77}$ & 0 & 0 & 0 & 1 \\
\hline$b_{22}$ & 0 & 0 & $1.3843 \cdot 10^{-5} \cdot T_{s}$ & $1.3843 \cdot 10^{-5} \cdot T_{s}$ \\
\hline$b_{41}$ & 0 & 0 & 0 & 6.8125 \\
\hline$f_{4}$ & 0 & -30 & -30 & -0.03707 \\
\hline$f_{5}$ & $T s$ & $T s$ & 0 & $T_{s}$ \\
\hline$f_{6}$ & 0 & 0 & $T_{s}$ & 0 \\
\hline$f_{7}$ & 0 & 0 & 0 & $T_{s}$ \\
\hline
\end{tabular}

Table 4

Parameter values

\begin{tabular}{|c||c|c|c|}
\hline & $i=20, i+2,32$ & $i=33$ & $i=34$ \\
\hline \hline$a_{44}$ & 0 & $-3.2116 \cdot 10^{-6} \cdot T_{s}$ & 0 \\
\hline$a_{55}$ & 0 & 0 & 0 \\
\hline$a_{66}$ & 1 & 1 & 1 \\
\hline$a_{77}$ & 1 & 0 & 0 \\
\hline$b_{22}$ & $1.3843 \cdot 10^{-5} \cdot T_{s}$ & $1.3843 \cdot 10^{-5} \cdot T_{s}$ & $1.3843 \cdot 10^{-5} \cdot T_{s}$ \\
\hline$b_{41}$ & 0 & 6.8125 & 0 \\
\hline$f_{4}$ & 0 & -0.03707 & 0 \\
\hline$f_{5}$ & 0 & 0 & 0 \\
\hline$f_{6}$ & $T_{s}$ & $T_{s}$ & 0 \\
\hline$f_{7}$ & $T_{s}$ & 0 & $T_{s}$ \\
\hline
\end{tabular}




\section{References}

[1] M. Arxer, L. M. Calleja, Hercules proyect. contributing to the development of hydrogen infrastructure, Journal of Power Sources 171 (1) (2007) 224-227.

[2] C. Chan, The state of the art of electric hybrid and fuel cell vehicles, in: Proceedings of the IEEE, Vol. 95, 2007, pp. 704-718.

[3] A. Burke, Batteries and ultracapacitors for electric, hybrid and fuel cell vehicles, in: Proceedings of the IEEE, Vol. 95, 2007, pp. 806-820.

[4] L. Guzella, A. Sciarretta, Vehicle Propulsion Systems: Introduction to Modeling and Optimization, Springer, 2005.

[5] J. Bernard, S. Delprat, F. Buechi, T. Guerra, Global optimization in the power management of a fuel cell hybrid vehicle (fchv), in: Proceedings of 2006 Vehicle Power and Propulsion Conference, 2006.

[6] M. Gielniak, Z. Shen, Power management strategy based on game theory for fuel cell hybrid electric vehicles, in: Proceedings of 2004 Vehicular Technology Conference, Vol. 6, 2004, pp. 4422-4426.

[7] A. Arce, Modelado y control de pilas de combustible en funcionamiento aislado e integrado en vehiculo de propulsion hibrida, Master's thesis, University of Seville (June 2007).

[8] F. Torrisi, A. Bemporad, HYSDEL-A tool for generating computational hybrid models, IEEE Trans. on Control Syst. Technol. 12 (2) (2004) 235-249.

[9] A. del Real, A. Arce, C. Bordons, Hybrid model predictive control of a two generator power plant integrating photovoltaic panels and fuel cell, in: Proceedings of 46th IEEE Conference on Decision and Control, 2007, pp. 5447-5452. 
[10] K. B. Wipke, M. R. Cuddy, S. D. Burch, Advisor 2.1: A user-friendly advanced powertrain simulation using a combined backward/forward approach, IEEE Transactions on Vehicular Technology 48 (6) (1999) 1751-1761.

[11] L. Gao, S. Liu, R. A. Dougal, Dynamic lithium-ion battery model for system simulation, IEEE Transactions on Components and Packaging Technologies 25 (3) (2002) 495-505.

[12] A. del Real, A. Arce, C. Bordons, Development and experimental validation of a PEM fuel cell dynamic model, Journal of Power Sources 173/1 (2007) 310-324.

[13] O. Sundström, A. Stefanopoulou, Optimum battery size for fuel cell hybrid electric vehicle. part I, Journal of Fuel Cell Science and Technology 4 (2007) 167-175.

[14] A. Arce, A. J. del Real, C. Bordons, Control heuristico de gestion de potencia de un vehiculo hibrido, in: Actas de las XXVIII Jornadas de Automatica, Huelva, 2007.

[15] V. Teofilo, L. Merrit, R. Hollandsworth, Advance lithium-ion battery charger, Aerospace and Electronic Systems Magazine, IEEE 12 (11) (1997) 30-36.

[16] M. Elias, K. Nor, N. Rahim, A. Arof, Lithium-ion battery charger for high energy application, in: Proc. 2003 Power Engineering Conference, 2003, pp. 283-288.

[17] P. Antsaklis, A brief introduction to the theory and applications of hybrid systems, in: IEEE Proc., Spec. Issue Hybrid Syst.: Theory Appl., Vol. 88, 2000, pp. 879-886.

[18] J. Lygeros, C. Tomlin, S.Sastry, Controllers for reachability specifications for hybrid systems, Automatica 35 (3) (1999) 349-370.

[19] W. Heemels, B. D. Schutter, A. Bemporad, Equivalence of hybrid dynamical models, Automatica 37 (7) (2001) 1085-1091.

[20] A. Bemporad, M. Morari, Control of systems integrating logic, dynamics and constraints, Automatica 35 (3) (1999) 407-427. 
[21] E. Camacho, C. Bordons, Model Predictive Control, 2nd Edition, Springer-Verlag, London, 2004.

[22] A. Arce, D. Ramirez, A. J. del Real, C. Bordons, Constrained explicit predictive control strategies for pem fuel cell systems, in: Proc. 46th IEEE Conference on Decision and Control, 2007. 